\title{
Carnets
}

Revue électronique d'études françaises de l'APEF

Première Série - 4 | 2012

(Res)sources de l'extravagance

\section{Chemins de traverse - Voies de travers}

\section{Guy Achard-Bayle}

\section{(2) OpenEdition \\ Journals}

Édition électronique

URL : http://journals.openedition.org/carnets/7132

DOI : 10.4000/carnets.7132

ISSN : 1646-7698

Éditeur

APEF

Édition imprimée

Date de publication : 1 janvier 2012

Pagination : 261-279

Référence électronique

Guy Achard-Bayle, "Chemins de traverse - Voies de travers », Carnets [En ligne], Première Série -

4 | 2012, mis en ligne le 20 juin 2018, consulté le 02 mai 2019. URL : http://journals.openedition.org/ carnets/7132 ; DOI : 10.4000/carnets.7132

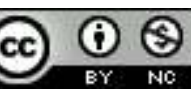

Carnets est mis à disposition selon les termes de la licence Creative Commons - Atribution - Pas d'utilisation commerciale 4.0 International. 


\title{
CHEMINS DE TRAVERSE - VOIES DE TRAVERS
}

\author{
GUY ACHARD-BAYLE \\ Université de Lorraine CREM-Praxitexte \\ guy.achardbayle@wanadoo.fr
}

\section{Résumé}

Dans cet article, que je conçois comme le rappel d'un parcours, j'exploite la notion d'extravagance au sens de sa composition étymologique: "extra-vagance"; autrement dit j'explore un domaine, à cheval entre littérature et linguistique, constitué de récits de métamorphose; j'essaie de montrer, dans le cadre d'une sémantique référentielle et textuelle, quels outils linguistiques permettent de réaliser littéralement ces phénomènes hors normes; je m'attache néanmoins à bien différencier la métamorphose, processus, de la métaphore, procédé, même si elles se croisent et s'influencent souvent dans les textes du corpus.

\begin{abstract}
In this article, I use and analyze the notion of extravagance in the sense of its etymological composition, "extra-vagance", in exploring a domain, between literature and linguistics, constituted by a corpus of stories telling metamorphosis, that is to say by texts or narrations which refer to unconventional, counterfactual cases or phenomena; but as a linguist, I observe and, I hope, I demonstrate that the language is able to represent them, sometimes with ambiguity. Nevertheless, I do make a great difference, even if they appear in the same context, between a metamorphosis, which is a process de re, and a metaphor, which is a procedure de dicto.
\end{abstract}

Mots-clés: linguistique textuelle, sémantique référentielle, référents évolutifs, de re vs de dicto Keywords: text linguistics, referential semantics, evolving referents, de re vs de dicto 
En termes d'extravagance, je voudrais m'interroger ici non seulement sur un domaine "hors norme" et "hors des sentiers battus", mais sur le parcours lui-même qui tout au long de la décennie écoulée (Achard-Bayle, 2001²009) m'a conduit à m'intéresser, et quasiment consacrer, à ce domaine.

Je voudrais donc me demander en quoi consiste, et au-delà, pourquoi faire une grammaire des métamorphoses. Me poser ces questions m'aidera, épistémologiquement parlant, à situer mon parcours dans le champ des sciences du langage et ses débats actuels.

II y en a divers, mais celui que j'ai privilégié et qui a été le déclencheur de mon second ouvrage (Achard-Bayle, 2008) repose sur - et même pose tout court - la question de la référence et de l'objectivité ( $v s$ la subjectivité) du sens, pour le dire assez grossièrement.

Même si je reviens dessus, pour la questionner plus tard, je prendrai ici la notion de référence pour acquise; autrement dit, très rapidement, mais suivant Quine (1990), je la considérerai comme la "relation mots-monde".

En somme les questions que je (me) pose aujourd'hui sont les suivantes: "Est-ce que cela a un sens de faire une grammaire des métamorphoses?" Autrement dit, dans les termes de sémantique référentielle tels que je les ai définis et adoptés: "Est-ce que cela m'apprend quelque chose du monde, sinon du langage, ou du moins des langues?" 1

Ces questions sont ambitieuses. II nous faut donc être prudents, car la métamorphose suscite de nombreuses conjectures, entre autres: "de la divinité à l'animalité", "du mythe au fantasme" (Belzane, 1990: 8 et 12). Ainsi, même si je parcours un domaine "hors des sentiers battus", je voudrais rester "terre à terre", si je peux dire, en privilégiant, suivant ma spécialité en linguistique, le niveau des textes.

Ainsi, dans la perspective épistémologique choisie, et pour répondre aux deux questions posées précédemment, je pourrais commencer par reprendre ces grands essais qui, de La Psychanalyse des contes de fées ${ }^{2}$ au Mythe de la métamorphose ${ }^{3}$, donnent un sens profondément humain, voire même naturel (naturaliste) à ces histoires à dormir debout... Je reviendrai sur B. Bettelheim, je dis en attendant un mot de P. Brunel et d'E. Renan:

\footnotetext{
1 Je travaille essentiellement sur des énoncés et des textes en français, mais parfois sur les originaux en allemand ou en anglais de grands classiques comme La Métamorphose ou L'étrange cas du Dr Jekyll et de Mr Hyde.

${ }^{2}$ Bettelheim (1976).

${ }^{3}$ Brunel (1974).
} 
Un homme est changé en âne, en oiseau, en poulpe, en pourceau, en cancrelat, en coyote. S'agit-il seulement d'histoires bonnes pour les enfants ou les fous [...]? Parmi tant de lecteurs [...] des Métamorphoses d'Ovide, il en est un qui sut saisir "dans cette suite délicieuse de mobiles et ravissantes images" un "rapport profond avec la nature", et dégager des mille énigmes qu'elles contiennent la question essentielle: "Difficiles enfantillages, que nous voulez-vous?"4

Ainsi, au-delà d'une réaction de mépris ou de rejet de ces phénomènes "hors normes", "irrationnels", "déviants" (ou "dé-viants", ce qui est une autre manière de dire: "extra-vagants"), on peut en effet comme Renan et Brunel se poser la question du bienfondé, du sens profond ${ }^{5}$ de ces "dé-viances", de ces "extra-vagances", qui instaurent des passerelles ou, mieux, des passages entre les espèces, et surtout entre les règnes; on peut encore considérer le passage des uns aux autres comme, entre autres, le signe d'un profond accord ou d'une étroite familiarité voire d'une continuité entre l'humain et la nature.

On peut ouvrir ici une perspective, avancer une hypothèse, à partir de la citation de Renan et du commentaire que l'on vient de faire, sur la continuité homme-nature, en citant, pour commencer, les travaux de l'ethnoscience tels qu'ils ont été exposés notamment dans l'anthologie de S. Arom et al. (1993); ou dans le même ordre d'idées, la Pensée sauvage de C. Lévi-Strauss (1962) qui montre diversement (notamment par des questions de catégorisation et de dénomination) que cette pensée dite sauvage repose sur l'analogie et l'homologie, ou, de manière plus générale, sur la motivation.

II faudrait encore sur ce sujet rappeler Les mots et les choses de M. Foucault (1966) où le philosophe présente les quatre "similitudes" qui forment, selon lui, "la trame sémiotique de la ressemblance au $\mathrm{XV}^{\mathrm{e}}$ siècle": la convenance, l'émulation, l'analogie, la sympathie.

Cette dernière notamment, "compensée" par l'antipathie (op. cit:: 63), "suscite le mouvement des choses dans le monde et provoque le rassemblement des plus distantes. Elle est le principe de mobilité" (je souligne dans les deux cas). Et Foucault de donner cet exemple à propos de la sympathie: "elle fait virer avec la courbe du soleil la grande fleur jaune du tournesol." J'en arrive ainsi à mes propres exemples et à mes premières analyses textuelles.

On trouve chez Ovide trois métamorphoses d'humains en végétaux qui impliquent Apollon, ou Phoebus, dieu du jour, mais aussi de la musique et des arts: avec Daphné qui ne l'aime pas mais qu'il veut attacher à lui; avec Clytie ou Clytié qu'il a, au contraire, délaissée pour sa sœur et qui se laisse mourir d'amour pour lui; avec le jeune Hyacinthe qui l'aime en

\footnotetext{
${ }^{4}$ Lettre d'E. Renan à G. Flaubert 8 sept. 1874, citée par P. Brunel, op. cit:: 7.

${ }^{5}$ Voir le titre de l'ouvrage dirigé par M. Colas-Blaise \& A. Beyaert-Geslin (2009): Le sens de la métamorphose.
} 
retour, ce qui provoque la jalousie de Zéphyr (ou Borée). D’où ces métamorphoses: Daphné est transformée en laurier; Clytié en héliotrope (on retrouve ici le motif du tournesol); Hyacinthe se retrouve dans la fleur du même nom (qui "depuis" porte son nom, nous dit le texte).

Du point de vue figuratif et épistémique on retrouve, dans ces trois métamorphoses, trois des quatre formes de la similitude de Foucault:

La sympathie - au sens où Foucault l'a définie pour le tournesol - se retrouve naturellement, si je peux dire, chez Clytié métamorphosée en héliotrope; elle ne cesse durant neuf jours de contempler le soleil dans sa course, se nourrit de rosée et de larmes, et se meurt ainsi d'amour non pas en perdant son anima, mais en l'occurrence en prenant racine:

Pour Clytié, bien que l'amour pût être l'excuse de son dépit, et son dépit celle de sa consolation, le dieu du jour ne la revit plus et désormais renonça aux joies de Vénus en sa compagnie. Depuis ce jour, la nymphe dépérit [...] Ses membres, dit-on, adhérèrent au sol; une pâleur livide les décolore en partie et les transforme en tiges exsangues; une partie reste rouge et une fleur toute semblable à la violette cache sa tête. Bien que retenue par sa racine, elle se tourne vers son cher Soleil, et même métamorphosée, elle lui garde son amour (Ovide, 1991: 118).

Je souligne dans cette séquence les marques linguistiques (noms, propre ou commun, pronoms dits personnels, dont un réfléchi, possessifs) qui forment une chaîne de référence ${ }^{6}$, autrement dit qui assurent la continuité de l'identité personnelle du référent désigné, en l'occurrence Clytié, quel que soit le changement radical qui l'affecte, "même métamorphosée" comme le dit le texte: on voit ainsi comment la langue, grâce aux outils et aux opérations linguistiques mis en œuvre, "colle" à la lettre du texte. Mais cela ne "colle" que jusqu'à un certain point, car, du fait du syntagme sa racine et du contact qui s'instaure entre ce syntagme et les désignations suivantes - elle se [tourne]... -, on a bien à faire en fin de compte (et de conte) à un être double ou hybride, même si dans la dichotomie entre tête et racine, c'est la première, c'est-à-dire la mémoire, donc l'âme, qui l'emporte.

Restent, parmi les exemples de métamorphoses et les types de similitude, deux cas à traiter: chez Daphné, on trouve la convenance, autrement dit l'assimilation ou la proximité (Foucault, op. cit.), sous la forme du laurier dont Apollon orne son front ${ }^{7}$; quant à l'émulation

\footnotetext{
${ }^{6}$ Cf. Schnedecker (1997).

${ }^{7}$ M. Turner, au début de son ouvrage sur l'esprit littéraire (1996: 7), considère ainsi le rôle de la parabole dans la formation "narrative" de la cognition: il s'agit, par là, de "mettre contre". Ou "jeter contre", suivant le TFLI: "Empr. au gr. Параßо入ń " comparaison, rapprochement, rencontre ", également terme de math. " parabole ", dér. de Парaßá $\lambda \lambda \omega$ " jeter quelque chose, jeter le long de, étendre le long de " (Пapá " auprès de " et $\beta a ́ \lambda \lambda \omega$ " lancer, jeter "). (http://atilf.atilf.fr/dendien/scripts/tlfiv5/visusel.exe?12;s=3593032875;r=1;nat=;sol=1;)
} 
ou la réduplication en miroir (Foucault, ibid.), on la trouve chez Hyacinthe sous la forme de la fleur qui portera dès lors son nom: "Devenu une fleur nouvelle, tu répéteras, par l'inscription de tes pétales, mes gémissements [et] il [Apollon] grave lui-même sur les feuilles, pour

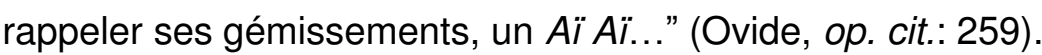

Après ce passage par la symbolique de la "pensée sauvage", je reviens à mes questions: "Est-ce que cela a un sens de faire une grammaire des métamorphoses? Est-ce que cela m'apprend quelque chose du monde?" Je pense bien entendu que oui et j'essaierai de le montrer en deux étapes dans les parties suivantes.

\section{Métamorphoses et visions du monde: Scala Naturæ, The Great Chain of Being}

À partir des années 2000, j'ai commencé à fréquenter les colloques de linguistique cognitive $^{8}$, et, de la sorte, à m'interroger sur la signification anthropologique des procédés de désignations analogiques dans les récits de métamorphose ou plus généralement dans ce que nous avons appelé en linguistique les contextes évolutifs; pour ma part (cf. AchardBayle, 2001b ; Silva, 2001), j'ai parlé de l'identité comme une idée du même.

C'est ainsi que je me suis intéressé au Literary Mind de M. Turner (1996), ou encore à toutes ces formes de pensée qui s'adressent aussi bien à notre imagination qu'à notre raison, d'où le titre de l'ouvrage de G. Lakoff \& M. Turner (1989): More than Cool Reason. La métaphore est ainsi cet instrument indispensable à une forme de pensée dite poétique mais en fait bien ordinaire, et non seulement quotidienne (cf. Lakoff \& Johnson, 1980), mais indispensable à la représentation de soi (de nous-mêmes: ourselves, cf. Lakoff \& Turner, op. cit.) et du monde.

Quant au Literary Mind de M. Turner, il fait du récit une forme originelle de la pensée littéraire, partant et parlant de la parabole comme d'un exemple de la pensée par projection ${ }^{9}$, en l'occurrence un récit projeté sur un autre ${ }^{10}$ : ainsi au début des Mille et Une Nuits, le récit

\footnotetext{
${ }^{8}$ Notamment Linguagem e cognição, Braga, mai 2000; Linguagem, cultura e cognição, Braga et ICLC'03 Logroño, juillet 2003.

${ }^{9}$ On se rapproche ainsi de l'étymologie exacte, rappelée en note ci-dessus.

${ }^{10}$ Cf. M. Le Guern (1981: 34) qui reprend P. Brunel et son Mythe de la métamorphose: "On peut dire de chaque récit de métamorphose que le fonctionnement de sa signification met en jeu un sens et une référence. [...] Le sens d'un mythe est la relation d'un signifiant avec un signifié, d'une histoire avec une réalité autre qu'elle signifie. Et si le mythe est irremplaçable, c'est qu'il donne le moyen d'exprimer ce que l'on ne saurait dire autrement, de révéler ce qui est caché si profondément que les mots ne permettent pas de l'atteindre [...] II est muthos,
} 
du Vizir à sa fille Shéhérazade, l'Histoire de l'âne, du bœuf et du laboureur, doit servir d'exemple (de dissuasion) à Shéhérazade ${ }^{11}$.

Revenons maintenant à la métaphore dans More than Cool Reason (Lakoff \& Turner, op. cit: 166 sq.). La théorie cognitive et culturelle de la métaphore (et des proverbes) que développent ces auteurs est la suivante: nous, membres d'une communauté linguistique, sociale, culturelle, avons un bagage ou un héritage partagé en matière d'expériences humaines - et de la place de l'humain dans le monde ou l'univers; c'est ainsi que les proverbes sont: - vérifier les normes (à cause de puis).

Instructions for understanding the nature of our being, the nature of people and situations we encounter, and our role in the universe. In each case, the instructions are addressed metaphorically. The presumption is that higher-order questions are answered in terms of lower-order descriptions [...] (op. cit.: 161)

What is taken for granted in proverbs is that we have a certain sense of the order of things, that we know a great deal about man's place in the universe [...] We understand proverbs as offering us ways of comprehending the complex faculties of human beings in terms of [...] other things. To do this, we use the Great Chain of Being.

The Great Chain of Being is a cultural model that concerns kinds of beings ant their properties and places them on a vertical scale with 'higher' beings and properties above 'lower' beings and properties [...] The Great Chain is a scale of forms of being -human, animal, plant, inanimate object- and consequently a scale of the properties that characterize forms of being - reason, instinctual behavior, biological function, physical attributes, and so on.

Commonly, the Great Chain of Being is taught as background to literature and the history of ideas, as essential to the understanding of the worldviews of classical authors like Plato and Aristotle, medieval authors like Dante and Chaucer, renaissance authors like Shakespeare, and even Augustan authors like Pope. But it is taught as if it somehow died out in the industrial age. On the contrary, a highly articulated version of it still exists as a contemporary unconscious cultural model indispensable to our understanding of ourselves, our world, and our language. (op. cit: 166-167).

J'ai cité ce long passage car il est fondamental pour comprendre l'orientation culturelle de la linguistique cognitive, telle que la défendent et illustrent nos auteurs (Rendre plus claire cette affirmation). J'en ferai maintenant un commentaire et me poserai à ce

mensonge; plus le mensonge est flagrant, plus la relation référentielle s'estompe, et plus fortement le sens s'impose."

${ }^{11}$ Nous reviendrons pour finir sur la question du récit. 
propos une question: en ce qui concerne le commentaire, je dirai que G. Lakoff \& M. Turner appliquent une grille de lecture antique aux mentalités ou aux Weltanschauungen contemporaines; ainsi, il y a dans leur théorie une réminiscence de la Scala Naturæ, reprise entre autres d'Aristote: ce modèle a prévalu, au moins, "depuis l'Antiquité jusqu'à la fin du XVIII siècle" (E. Mayr, 1982, tome 1: 38), même si c'est sous la forme de ce qu'A. Lovejoy (1936) appelle The Great Chain of Being ${ }^{12}$.

En ce qui concerne maintenant la question, je me demanderai quelle place est faite au langage dans cette théorie de l'esprit littéraire. Je pense l'avoir déjà montré par un premier récit de métamorphose (ci-dessus Clytié), mais l'exemple était assez bref: je voudrais par conséquent reprendre la démonstration, et particulièrement ma démonstration de 2001(b) qui concernait l'identité et l'idée du même, c'est-à-dire l'identité comme l'idée du même; je vais en fait comparer diverses métamorphoses des Mille et Une Nuits, et en comparer les effets (supposés) sur le lecteur. Pour clore cette partie, je ferai le bilan de toutes nos premières analyses textuelles, en revenant sur Ovide, à la lumière des Mille et Une Nuits.

M'étant avancée dans une quatrième cour, j'y vis en face un très-beau bâtiment dont les fenêtres étaient fermées d'un treillis d'or massif. Je jugeai que c'était l'appartement de la reine. J'y entrai. II y avait dans une salle plusieurs eunuques noirs pétrifiés. Je passai ensuite dans une chambre très-richement meublée, où j'aperçus une dame aussi changée en pierre. Je connus que c'était la reine à une couronne d'or qu'elle avait sur la tête et à un collier de perles très-rondes et plus grosses que des noisettes. Je les examinai de près; il me parut qu'on ne pouvait rien voir de plus beau $[\ldots]$

II y a trois ans et quelques mois qu'une voix bruyante se fit tout à coup entendre par toute la ville si distinctement, que personne ne perdit une de ces paroles qu'elle dit: "Habitants, abandonnez le culte de Nardoun et du feu; adorez le Dieu unique qui fait miséricorde".

La même voix se fit ouïr trois années de suite, mais personne ne s'étant converti, le dernier jour de la troisième, à trois ou quatre heures du matin, tous les habitants généralement furent changés en pierre en un instant, chacun dans l'état et la posture où il se trouva. Le roi mon père éprouva le même sort: il fut métamorphosé en une pierre noire, tel qu'on le voit dans un endroit de ce palais... ${ }^{13}$

\footnotetext{
${ }^{12}$ Sans doute G. Lakoff \& M. Turner lui empruntent-ils l'expression qu'ils utilisent également.

${ }^{13}$ Mille et Une Nuits extraits $1 \& 2$ (Histoire de Zobéide, trad. Antoine Galland: 212; je souligne à deux reprises).
} 
Dans ces deux premières métamorphoses, l'identité des personnages métamorphosés, c'est-à-dire leur identité première ou initiale, est maintenue grâce à la ressemblance; ou plus précisément grâce à la reconnaissance, au sens de $\mathrm{P}$. Ricœur (2004): il s'agit d'un procédé d'identification qui permet de substituer un souvenir à ce qui est, d'abord, vu ou aperçu; ainsi dans le premier cas, une dame, d'abord inconnue donc indéfinie, devient-elle ou plutôt redevient-elle la reine, reconnue ou redéfinie comme telle; dans le second cas, on continue de voir le roi, fût-il changé en une pierre noire...

Il y a une différence néanmoins: dans le second cas, le roi n'est jamais perdu de vue, si l'on peut dire, la chaîne de référence étant maintenue, telle que je la souligne dans l'extrait suivant- vous n'avez pas souligné!: "Le roi mon père éprouva le même sort: il fut métamorphosé en une pierre noire, tel qu'on le voit..."

Ainsi, la différence linguistique ou textuelle entre les deux métamorphoses, ou entre les deux textes qui les relatent, est le maintien ou non de la chaîne de référence: dans le premier texte, elle a été rompue, ou n'est reconstituée qu'après que le personnagenarrateur ${ }^{14}$ a rétabli (par reconnaissance) le lien d'identité dame $=$ reine:

Je jugeai que c'était l'appartement de la reine. J'y entrai. II y avait dans une salle plusieurs eunuques noirs pétrifiés. Je passai ensuite dans une chambre trèsrichement meublée, où j'aperçus une dame aussi changée en pierre. Je connus que c'était la reine...

Voyons maintenant deux autres métamorphoses (il va s'agir en fait ici de deux traductions d'une même histoire):

Je revins chez moi et trouvais mon épouse plongée dans le sommeil. Je jetai sur elle quelques gouttes d'eau en lui ordonnant de se transformer en mule. Aussitôt elle se métamorphosa. Je la saisis par la crinière et la fis descendre au bas de la maison où je l'attachais. Le lendemain je lui passais - vérifier s'il s'agit d'un imparfait! - un mors, la sanglai et la bâtai. Je me munis d'une cravache en cuir et, depuis, je la monte chaque jour. C'est cet animal que tu vois aujourd'hui de tes propres yeux, souverain et maître des démons.

Le vieillard se tourna vers la mule et lui demanda si ce qu'il venait de raconter était vrai, elle opina de la tête." 15

[...] je la ["mon épouse"] trouvais, l'aspergeais avec l'eau, et je dis: "Sors de cette forme-ci et deviens l'image d'une mule!" et à l'heure même elle devint une mule. Et c'est elle-même que tu vois là de ton propre œil, ô sultan et chef des rois des genn!"

\footnotetext{
${ }^{14}$ Dans les termes de G. Genette (1972), il est à la fois voix et vision...

${ }^{15}$ Histoire du troisième cheikh, trad. Mardrus, tome 1: 20.
} 
Alors le genni se tourna vers la mule et lui dit: "Est-ce vrai cela?" Et elle se mit à hocher la tête et dit par signes: "Oh oui! oh oui! cela est vrai. ${ }^{16}$

La différence entre ces deux textes et les deux précédents tient dans le fait qu'ici l'identité (initiale) n'est plus maintenue, du moins sous la forme nominale que lui confèrent les désignations; ou encore, dans le fait que la chaîne de référence est définitivement rompue, car, au bout du compte, c'est la mule qui a pris la place de l'épouse.

Néanmoins le comique, ou même "l'illusion comique", veut que la langue permette de redésigner celles-ci de la même manière, grâce aux pronoms; ainsi les deux traductions peuvent-elles faire sourire dans la mesure où elle s'applique indifféremment à l'une ou l'autre: l'épouse et la mule! Le double effet, d'"illusion comique", est particulièrement saisissant dans la traduction Bencheikh \& Miquel: "Aussitôt elle se métamorphosa. Je la saisis par la crinière et la fis descendre au bas de la maison où je l'attachais..."

Dans cette traduction, la chaîne est rompue au vu des prédicats: si c'est bien l'épouse qui se métamorphose, c'est la mule que le personnage-narrateur saisit par la crinière, fait descendre, attache, etc. Pour autant, par l'attraction du genre grammatical qui se maintient sous la forme elle, et par l'absence d'antécédent nominal immédiat (du type la mule), on a ou on peut avoir l'impression que la chaîne de référence n'est pas rompue: d'où l'effet comique.

Dans la seconde traduction (Mardrus), l'illusion et le comique qui en résulte sont peutêtre plus étudiés ou poussés encore, dans la mesure où la forme pronominale choisie, ellemême, met l'accent sur l'identité de soi avec soi, sans néanmoins résoudre l'énigme: qui de la femme ou de la mule est visée par l'expression référentielle elle-même? Autrement dit, il reste difficile de décider quel est le bon antécédent des deux qui se font concurrence au féminin singulier: "[...] à l'heure même elle devint une mule. Et c'est elle-même que tu vois là de ton propre œil..."

On peut tirer une leçon de cette première phase d'analyse et d'interprétation de récits de métamorphose, d'Ovide aux Mille et Une Nuits: il me semble que nous sommes enclins à maintenir sinon le lien coréférentiel, du moins une certaine continuité d'identité (fût-ce au prix

\footnotetext{
${ }^{16}$ Mille et Une Nuits extraits 3 \& 4 (Histoire du troisième vieillard, trad. Bencheikh \& Miquel, tome $1: 67$ )
} 
d'une certaine confusion) dans le cas où la métamorphose concerne, dans la scala naturæ, la Grande Chaîne, un humain et un animal - voire un végétal chez Ovide.

Par contre avec le minéral, les opérations métamorphiques ont des résultats divers:

Chez Galland, la forme de la reine, conservée comme statue, ou même la puissance de la mémoire pour le roi, dont on ne sait pas si la pierre noire qu'il est devenu est une figuration, maintiennent l'identité; mais au-delà de la figuration donc de la ressemblance, il s'agit dans les deux cas de formes, et de la force, du souvenir.

Chez Ovide, il y a diverses métamorphoses d'humain en minéral (ou en élément, disons, "inorganique"): Battus en rocher, Aglauros en statue, Byblis en source; mais dans tous les cas, que je souligne, les chaînes de référence sont clairement rompues:

Le petit-fils d'Atlas [Mercure] [...] change le cœur parjure [Battus] en une dure pierre, qui aujourd'hui encore est appelée l'Indicatrice; et un vieux renom d'opprobre pèse sur cette pierre bien innocente.

Elle [Aglauros ne tenta pas de parler; l'eût-elle tenté, la voix ne passait plus. Son cou était déjà pétrifié, son visage durci: ce n'était plus qu'une statue exsangue, assise. Et la pierre n'était même pas blanche: son âme l'avait salie.

[...] ainsi Byblis, descendante de Phœbus, consumée par ses propres larmes, est changée en une source qui, aujourd'hui encore, dans ces vallées, garde le nom de sa maîtresse et sort de terre au pied d'une yeuse noire. (Ovide, op. cit.: 84 , 87 et 248)

Par comparaison, les Mille et Une Nuits privilégient, à travers le souvenir, le modèle ou "l'idée de l'image". C'est "l'absent du présent" de la représentation".

Je tire maintenant une autre leçon (qui sera développée dans la partie suivante): la langue n'aide pas vraiment à débrouiller les "obscurités" du monde, qui résultent des continuités, et donc des ambiguïtés entre les êtres de la Grande Chaîne dont nous parlions... Mais précisément, si cela ajoute à l'illusion, voire à la confusion, on peut en tirer également la leçon qu'il n'existe pas une langue propre à la fiction.

On va ainsi en faire la démonstration, en étudiant le rôle de la métaphore dans la construction de l'identité hybride ou ambiguë: de la métaphore à la métamorphose; pour finir,

\footnotetext{
17 Je me réfère ici aux travaux de G. Genette (1994) et, surtout, de C. Ginzburg (2001) qui écrit au début de son ch. 3 Représentation (op. cit:: 73): "on parle beaucoup dans les sciences humaines, et depuis longtemps, de "représentation", succès sans doute dû à l'ambiguïté du terme. D'un côté, la représentation tient lieu de la réalité qu'elle représente et, par conséquent, évoque une absence; de l'autre, elle rend visible la réalité qu'elle représente et suggère donc une présence. Mis cette opposition pourrait aisément être retournée: dans le premier cas, la représentation est présente, ne serait-ce qu'au titre de substitut; dans le second, elle rappelle seulement une réalité absente qu'elle entendait pourtant représenter*. [ ${ }^{\star} E n$ note, p. 203: Robert Chartier, Le monde comme représentation, Annales E.S.C., 6, 1989, pp. 1505 1520]
} 
en conclusion, nous procéderons à un autre élargissement, en nous attachant à la question plus générale du sens et de la fiction.

\section{De la métaphore à la métamorphose}

J'avais assez longuement traité la question dans mon premier ouvrage (2001a: ch. 8). Ceci dit, mes recherches en linguistique cognitive, entreprises depuis, m'ont conduit à revenir sur cette question, notamment à la lumière de la Great Chain of Being, qui justifie l'étude continue des deux procédures.

Mais je dois préciser avant tout ce que j'entends par "procédures", et la différence que je fais entre processus métamorphique et procédé métaphorique: d'un point de vue logique, l'un est de re, l'autre de dicto; l'un relève de ce que j'ai appelé (op. cit.: 231-232) la recatégorisation, l'autre de la reclassification: la recatégorisation - avec changement de marque d'identité nominale - a lieu après l'accomplissement effectif d'une métamorphose, notamment un changement d'espèce; tandis que la reclassification est un changement de désignation nominale (sous la forme d'une anaphore infidèle) sans effet métamorphique sur l'identité ${ }^{18}$. Voyons cela par l'exemple:

Mélion entre alors dans la forêt, ôte ses vêtements et reste nu, enveloppé seulement de son manteau. Sa femme le touche, tout nu, avec l'anneau: il devient alors un loup grand et fort.

Le loup court vers l'endroit où il a vu le cerf couché... (Le lai de Mélion, éd. citée: 269)

On a ici un cas tout à fait caractéristique de recatégorisation. Le SN indéfini attributif un loup devient un SN défini, référentiellement autonome: le loup, qui instaure une nouvelle chaîne de référence: Mélion est devenu un loup; le loup remplace Mélion. Cette opération linguistique est une forme d'enregistrement écrit, textue ${ }^{19}$ de la métamorphose, autrement dit du processus métamorphique.

Or, on ne peut voir de la même manière un SN attributif métaphorique devenir un SN référentiel; ainsi avec: "L'homme est un loup pour l'homme", il sera difficile de trouver une suite telle que: "Et le loup est aussi un con pour les loups"20.

Par contre on pourrait avoir en reprise anaphorique un SN démonstratif: "Et ce loup..."21

\footnotetext{
${ }^{18}$ Voir F. Corblin (1995).

${ }^{19}$ Sur l'enregistrement textuel, voir Karttunen (1976).

20 J'adapte ici, faute d'occurrence authentique, cet exemple trouvé sur Internet: "L'homme est un loup pour l'homme et un gros con pour le loup." (http://www.monde-solidaire.org/spip/spip.php?article2567)- Citez le site.

${ }^{21}$ C'est l'un des rôles fondamentaux du SN démonstratif (par opposition au SN déf.): cf. G. Kleiber (1994).
} 
Voyons un second exemple d'opposition métamorphose vs métaphore, que je souligne, chez La Fontaine:

Les voilà devenus ours, lions, éléphants [...] Ulysse du lion court à l'ours... (Les Compagnons d'Ulysse, Fables, XII, 1, vers 37 et 65)

Toute sa personne velue / Représentait un ours, mais un ours mal léché... (Le Paysan du Danube, Fables, XI, 7, vers 12-13)

L'ours de la seconde fable n'est qu'une image; il n'est donc pas destiné à remplacer dans le texte le Paysan du Danube, qu'il figure ou représente au sens de Ginzburg (op. cit. et supra).

Donc la Grande Chaîne des $\hat{E}_{\text {tres }}{ }^{22}$, telle que les métamorphoses l'illustrent, parmi d'autres sémiotisations du monde ${ }^{23}$, me conduit toujours à mettre en parallèle, suivant encore divers mouvements de proximité, donc par appariement, approximation, la métamorphose et la métaphore.

Du point de vue narratologique, ce qui m'intéresse, alors, c'est que dans les contextes évolutifs les métaphores ou les assimilations animalières précèdent, assez régulièrement, les métamorphoses (notamment de l'humain en animal); voyons-le ici par l'exemple d'Hugues le loup ${ }^{24}$.

[L'étudiant Fritz et son ami braconnier Gédéon séjournent pour chasser au château du comte Nideck. Celui-ci est un loup-garou, ce qui veut dire que dans certaines séquences, il est, y compris nominalement, un loup. En attendant voici le récit de la première rencontre, son premier portrait qui anticipe bien la métamorphose (Fritz est à la fois la voix et la vision du récit)]:

Dès le premier instant, je fus saisi de l'étrange physionomie du seigneur de Nideck, et, malgré toute l'admiration respectueuse que venait de m'inspirer sa fille, je ne pus m'empêcher de me dire: “C'est un vieux loup!" En effet, cette tête grise à cheveux ras [...] singulièrement allongée, [...], l'étroitesse du front au sommet, sa largeur à la base, [...], la barbe courte et drue [...] tout dans cet homme me fit frémir, et des idées bizarres sur les affinités animales me traversèrent l'esprit..

\footnotetext{
${ }^{22}$ Dans une perspective logicodiscursive, on peut la rapprocher de ce que M. Foucault (op. cit.) appelle, lui, une "prose du monde".

${ }^{23} \mathrm{Je}$ reprends là un des termes-clés du colloque Métamorphoses de Luxembourg 2007: voir M. Colas-Blaise et A. Beyaert-Geslin (2009).

${ }^{24}$ Erckmann-Chatrian, in Contes fantastiques (éd, citée: 31 et 77-78). Je souligne.
} 
[À la fin du conte, Fritz, qui a vu et poursuivi un loup dans les bois, "redéfinit" le comte] Le comte de Nideck, accroupi sur son lit, les deux bras en avant, la tête basse, inclinée sous les tentures rouges, les yeux étincelants, poussait des hurlements lugubres!

- Le loup... c'était lui!...

Ce front plat, ce visage allongé en pointe, cette barbe roussâtre, hérissée sur les joues, cette longue échine maigre, ces jambes nerveuses, la face, le cri, l'attitude, tout, tout révélait la bête fauve cachée sous le masque humain!

La différence entre ce cas et la sentence de Hobbes: Homo homini lupus, c'est que l'équation attributive qui s'établit entre deux SN, l'un humain, l'autre animal, se fait différemment:

- Dans "L'homme est un loup [pour l'homme]", l'homme a beau être un loup, il reste un homme: il l'est attributivement et figurativement; un peu comme le Paysan du Danube est un ours.

- Dans "Le loup... c'était lui!", d'abord, on a l'occurrence inverse des SN, l'animal avant l'humain, mais surtout, on a affaire à deux désignations référentielles non coréférentielles: autrement dit lui n'est pas attributif et désigne un être différent du loup qui précède - du moins si l'on considère la succession des identités dans le temps et la réversibilité des métamorphoses en loup-garou: ce qui, dans la fable de La Fontaine, n'est pas le cas d'un des compagnons d'Ulysse, qui lui refuse de redevenir homme.

Je tire donc de cette nouvelle phase de démonstrations la leçon que l'étude comparée des deux procédures justifie l'approche référentielle que nous avons choisie: elle consiste, quel que soit le lien mots-monde qui définit la référence, à distinguer d'un point de vue logicodiscursif les faits de re des faits de dicto ${ }^{25}$.

Je voudrais le montrer par un dernier exemple (je souligne), qui vaudra doublement pour la démonstration en cours: d'abord parce que, même s'il s'agit d'un texte poétique, les deux procédures sont clairement distinguées; mais ensuite parce qu'elles apparaissent dans

\footnotetext{
${ }^{25}$ Cf., pour revenir au corpus, M. Le Guern (art. cité: 35): "On peut dire de chaque récit de métamorphose que le fonctionnement de sa signification met en jeu un sens et une référence. La référence, c'est la relation des mots avec les choses; dans le cas de la métamorphose, c'est la relation des mots avec ce qui existe, et peu importe, au fond, que cette existence ait une réalité physique ou qu'elle appartienne à un monde imaginaire..."
} 
l'ordre inverse à celui qui prévaut dans les récits de métamorphoses comme Hugues le loup, où, pour des raisons spectaculaires évidentes, la métaphore préfigure...

Le Papillon

Lorsque le sucre élaboré dans les tiges surgit au fond des fleurs, comme des tasses mal lavées, - un grand effort se produit par terre d'où les papillons tout à coup prennent leur vol.

Mais comme chaque chenille eut la tête aveuglée et laissée noire, et le torse amaigri par la véritable explosion d'où les ailes symétriques flambèrent,

Dès lors le papillon erratique ne se pose plus qu'au hasard de sa course, ou tout comme.

Allumette volante, sa flamme n'est pas contagieuse. Et d'ailleurs, il arrive trop tard et ne peut que constater les fleurs écloses. N'importe: se conduisant en lampiste, il vérifie la provision d'huile de chacune. II pose au sommet des fleurs la guenille atrophiée qu'il emporte et venge ainsi sa longue humiliation amorphe de chenille au pied des tiges.

Minuscule voilier des airs maltraité par le vent en pétale superfétatoire, il vagabonde au jardin

(Ponge, 1971)

\section{Pour finir}

Je termine en bouclant, c'est-à-dire en revenant à la citation d'E. Renan: "Difficiles enfantillages...". Pour ma part, je fais de la propriété de ces histoires "à dormir debout", le fondement de ma recherche. Les (récits de) métamorphoses servent et m'ont servi en effet de laboratoire de l'identité26: ces cas contrefactuels (au sens où l'entendent les philosophes analytiques quand ils parlent de puzzling $\operatorname{cases}^{27}$ ) ont une valeur heuristique; mais je crois aussi, suivant maintenant B. Bettelheim, qu'on peut facilement donner à cette valeur une dimension épistémique et sémiotique qui va au-delà de la découverte de soi: "Les contes de fées disent qu'il vient un moment où nous devons apprendre ce que nous ne savions pas jusque-là, autrement dit, en termes de psychanalyse, un moment où nous devons cesser de refouler notre sexualité" (Bettelheim, op. cit.: 404).

Néanmoins, dans les contes de fées comme dans les récits de métamorphoses, les premiers incluant souvent les seconds, c'est bien l'humain au sens large, et pas seulement "en termes de psychanalyse", qui est en jeu et mis en scène, l'un passant par l'autre: la

\footnotetext{
${ }^{26}$ Repris de P. Ricœur (1990).

${ }^{27}$ Voir après P. Ricœur, S. Ferret (1996).
} 
représentation, et elle seule, donne une dimension palpable, démonstrative à l'interrogation "onto-logique".

En outre, si l'humain est, entre autres, une des conditions et donc une des caractéristiques du récit - ce que J.-M. Adam dans divers ouvrages $(1984,1985)$ consacrés au texte narratif et au récit appelle, avec C. Bremond (1966), "l'implication d'intérêt humain"28 -, il faut alors noter la convergence entre le "schéma de la métamorphose mythique [avec ses] forme initiale, transformation, forme finale" (selon M. Le Guern, 1981: 34) et le récit ou la séquence narrative tels que les a définis Aristote (Poétique), puis tels qu'ils ont été redéfinis par Paul Ricœur (1983): comme une transformation d'état entre situation initiale et situation finale $e^{29}$.

Je termine en passant de l'heuristique à l'herméneutique grâce une fois de plus à $P$. Ricœur; ainsi, je reviens moi-même à mon point de départ, la référence.

Si la fiction est le fondement de la logique des mondes possibles, où (suivant P. Ricœur $1986^{30}$ ) la littérature nous projette, alors il faut bien qu'un monde de référence existe $^{31}$, et que ces mondes possibles le reflètent ou le rejettent. Et si la continuité, ou sinon la cohérence, est assurée, c'est parce qu'en passant de l'un aux autres, la langue ne change pas. Je redonne ainsi, et pour tout à fait terminer, la parole à P. Ricœur:

Avec la phrase, le langage est orienté au-delà de lui-même: il dit quelque chose sur quelque chose. Cette visée d'un référent du discours est rigoureusement contemporaine de son caractère d'événement et de son fonctionnement dialogal. Elle est l'autre versant de l'instance de discours. L'événement complet, c'est non seulement que quelqu'un prenne la parole et s'adresse à un interlocuteur, c'est aussi qu'il ambitionne de porter au langage et de partager avec autrui une expérience nouvelle. C'est cette expérience qui, à son tour, a le monde pour horizon [...] le langage ne constitue pas un monde pour lui-même. II n'est même pas du tout un monde. Parce que nous sommes dans le monde et affectés par des situations, nous tentons de nous y orienter sur le

\footnotetext{
${ }^{28}$ Voir aussi H. Naïs (1981: 24) qui cite le Dictionnaire du bon français de M. Dupré: "la métamorphose s'applique aux humains, la transmutation aux choses, et métamorphoser est l'œuvre des Dieux."

${ }_{29}$ Voir aussi les "prédicats transformés" chez J.-M. Adam (1984: 88), à mettre en relation avec ce que M. Charolles \& J. François (1988) appellent les prédicats transformateurs.

30 "[...] ce qui est en effet à interpréter dans un texte, c'est une proposition de monde, d'un monde tel que je puisse l'habiter pour y projeter un de mes possibles les plus propres“ (Ricœur, 1986, rééd. 1998: 128).

${ }^{31}$ Cf. le "monde de ce qui est" chez R. Martin (1983).
} 
mode de la compréhension et nous avons quelque chose à dire, une expérience à porter au langage et à partager.

Telle est la présupposition ontologique de la référence, présupposition réfléchie à l'intérieur du langage lui-même comme un postulat dénué de justification immanente. Le langage est pour lui-même de l'ordre du Même; le monde est son Autre. L'attestation de cette altérité relève de la réflexivité du langage sur lui-même, qui, ainsi, se sait dans l'être afin de porter sur l'être.

Cette présupposition ne relève ni de la linguistique, ni de la sémiotique; au contraire, ces sciences rejettent par postulat de méthode l'idée d'une visée intentionnelle orientée vers l'extralinguistique. Ce que je viens d'appeler l'attestation ontologique doit leur apparaître, une fois posé leur postulat de méthode, comme un saut injustifiable et inadmissible. De fait, cette attestation ontologique resterait un saut irrationnel si l'extériorisation qu'elle exige n'était pas la contrepartie d'une motion préalable et plus originaire, partant de l'expérience d'être dans le monde et dans le temps, et procédant de cette condition ontologique vers son expression dans le langage ${ }^{32}$.

${ }^{32}$ Ricœur, 1983, § Narrativité et référence, pp. 147-148 de la rééd. 1991. 


\section{Bibliographie}

Corpus:

ErCKMANn-Chatrian. Hugues-le-Loup in Contes fantastiques complets. Paris: Néo, 1987.

LA FONTAINE. Fables. In Jean-Pierre Collinet (éd.) (1991), CEuvres poétiques complètes I. Paris: Gallimard, coll. La Pléiade.

LES MILLE ET UNE NUITS, Contes arabes, vol. 1. Éd. Jean Gaulmier, trad. Antoine Galland (en X vol. 1704-1712). Paris: Garnier-Flammarion, coll. GF, 1965.

LES MILLE ET UNE NUITS, vol. 1. Trad. Jamel Eddine Bencheikh \& André Miquel, Paris: Gallimard, 1991, rééd. coll. Folio, 2005.

LES MILLE ET UNE NUITS, vol. 1. Trad. Joseph-Charles Mardrus, 1899-1904. Rééd Paris: Laffont: Bouquins, 2006.

LE LAI DE MELION. In Alexandre Micha (éd.), Les lais féériques des XIlème et XIIlème siècles, éd. bilingue. Paris: Flammarion, coll. GF, 1992.

OVIDE. Métamorphoses. Trad. Joseph Chamonard (1966, 1991). Rééd. Paris: Flammarion, coll. G.-F., 1991.

PONGE, Francis (1942). Le Papillon in Le Parti-pris des choses. Paris: Gallimard, rééd. Coll. Poésie, 1971.

Études:

ACHARD-BAYLE, Guy (2001a). Grammaire des métamorphoses. Référence, Identité, Changement, Fiction. Bruxelles: Duculot De Boeck, coll. Champs linguistiques-Recherches.

ACHARD-BAYLE, Guy (2001b). "Les référents évolutifs: identité et idée du même". In António Soares da Silva (ed.), Actas do Encontro Internacional Linguagem e Cognição: A Perspectiva da Linguística Cognitiva, 22-24 mai 2000. Braga: Associação Portuguesa de Linguística e Faculdade de Filosofia, pp. 277-304.

Achard-BAyle, Guy (2008). Les Réalités conceptuelles. Identité et/en Fiction. Metz: Publications de I'Université Paul Verlaine, coll. Recherches textuelles 8.

ACHARD-BAYLE, Guy (2009). "Idée fixe et double vue. Retour sur une Grammaire des métamorphoses". In Marion Colas-Blaise \& Anne Beyaert-Geslin (éds), Le sens de la métamorphose. Limoges: PULIM, pp. 31-47. ${ }^{33}$

ADAM, Jean-Michel (1984). Le récit. Paris: Presses Universitaires de France.

ADAM, Jean-Michel (1985). Le texte narratif. Paris: Nathan.

Aristote (Magnien Michel éd. 1990). Poétique. Paris: Librairie Générale Française, Le Livre de Poche.

AROM, Shima et al. interrogés par SCHEPS, Ruth (1993). La science sauvage. Des savoirs populaires aux ethnosciences. Paris: Editions du Seuil, coll. Points-Sciences.

BAUSTERT, Raymond, RaUS, Tonia \& WILHELM Frank (éds 2009). Cigare-Poisson. La métamorphose: un procédé à l'œuvre, Actes de Métamorphoses. Colloque international en littérature et sémiotique, Université de Luxembourg 10-12 mai 2007. Metz: Publications de l'Université Paul Verlaine, coll. Recherches en littérature 2.

Belzane, Guy (1990). La Métamorphose. Ovide, Perrault, Hugo, Michaux. Paris: Quintette.

BetTelheim, Bruno (1976, trad. fr. et rééd 1990). Psychanalyse des contes de fées. Paris: Hachette, coll. Pluriel.

\footnotetext{
${ }^{33}$ Voir la suite in R. Baustert et al. (2009).
} 
Bremond, Claude (1966). "La logique des genres narratifs". In: Communications, n8, pp. 60-76.

BREMOND, Claude (1973). Logique du récit. Paris: Éditions du Seuil.

BRUNEL, Pierre (1974, rééd. 2004). Le Mythe de la métamorphose. Paris: José Corti, coll. Les Massicotés.

CHAROLLES, Michel \& FRANÇOIS, Jacques (1998). Les prédications transformatrices et leurs patients: reprises pronominales et changements. Université Nancy 2: Cahier de recherche linguistique $n^{\circ}$ 11.

CHARTIER, Robert (1989). "Le monde comme représentation”. In Annales E.S.C., n 6, pp. 15051520.

ColAs-Blaise, Marion \& BeYAERT-Geslin, Anne (éds 2009), Le sens de la métamorphose. Limoges: PULIM.

CONTE, Maria-Elisabeth (a cura di 1981), La linguistica testuale. Milan: Feltrinelli.

CORBLIN, Francis (1995). Les formes de reprise dans le discours. Rennes: Presses Universitaires.

DuBoIs, Claude-Gilbert (éd. 1981), Poétiques de la métamorphose. Saint-Étienne: Publications de l'Université.

FERRET, Stéphane (1996). Le Bateau de Thésée. Le problème de l'identité dans le temps. Paris: Les Éditions de Minuit.

FOUCAULT, Michel (1966). Les Mots et les choses. Paris: Gallimard.

GENETTE, Gérard (1972). Figures III. Paris: Éditions du Seuil.

GinZBURG, Carlo (1998, trad. fr. 2001). À Distance. Paris: Gallimard.

KARTTUNEN, Lauri (1976, trad. it. 1981). "Referenti testuali". In Maria-Elisabeth Conte (a cura di), La linguistica testuale. Milan: Feltrinelli, pp. 121-147.

KLEIBER, Georges (1994). Anaphores et pronoms. Louvain-la-Neuve: Duculot.

LAKOFF, George \& JOHNSON, Mark (1980, trad. fr. 1985). Les métaphores dans la vie quotidienne. Paris: Les Éditions de Minuit.

LAKOFF, George \& TURNER, Mark (1989). More than Cool Reason. A Field Guide to Poetic Metaphor. Chicago: The University of Chicago Press.

LE GUERN, Michel (1981). "La métamorphose poétique: essai de définition". In Claude-Gilbert Dubois (éd.), Poétiques de la métamorphose. Saint-Étienne: Publications de l'Université, pp. 27-36.

LeVI-STRAuss, Claude (1962, rééd. 1990). La pensée sauvage. Paris: Presses-Pocket, coll. Agora.

LOVEJOY, Arthur (1936, reprint 2005). The Great Chain of Being: A Study of the History of an Idea. New York: Harper \& Row, paperback.

MAYR, Ernst (1982, trad. fr. et rééd. 1995). The Growth of Biological Thought. Diversity, Evolution and Inheritance. Paris: Le Livre de Poche, coll. Références, 2 vol.

MARTIN, Robert (1983). La logique du sens. Paris, Presses Universitaires de France.

NAïs, Hélène (1981). "Pour une notice lexicographique sur le mot métamorphose". In Claude-Gilbert Dubois (éd.), Poétiques de la métamorphose. Saint-Étienne: Publications de l'Université, pp. 1525.

QUINE, Willard van Orman (1990 trad. fr. 1993). La poursuite de la vérité. Paris: Éditions du Seuil.

RICCEUR, Paul (1983, rééd. 1991). Temps et récit, 1. L'intrigue et le récit historique. Paris: Éditions du Seuil, coll. Points.

RICœEUR, Paul (1986, rééd. 1998). Du texte à l'action. Essais d'herméneutique II. Paris: Éditions du Seuil, coll. Points (voir particulièrement: "Qu'est-ce qu'un texte?", pp. 153-203).

RICOEUR, Paul (1990). Soi-même comme un autre. Paris: Éditions du Seuil.

SILVA, Antonio Soares da (ed.) (2001), Actas do Encontro Internacional Linguagem e Cognição: A Perspectiva da Linguística Cognitiva, 22-24 mai 2000. Braga: Associação Portuguesa de Linguística e Faculdade de Filosofia. 
SCHNEDECKER, Catherine (1997). Noms propres \& chaînes de référence. Paris: Klincksieck \& Metz: Publications de l'Université, coll. Recherches linguistiques, $\mathrm{n}^{\circ} 21$.

TURNER, Mark (1996). The Literary Mind. The origins of Thought and Language; Oxford: Oxford University Press. 